Exp. Anim. 56(5), 355-362, 2007

\title{
Effect of Insulin Therapy on Renal Changes in Spontaneously Diabetic Torii Rats
}

\author{
Takeshi OHTA ${ }^{1)}$, Kenichi MATSUI'), Katsuhiro MIYAJIMA'2), \\ Tomohiko SASASE ${ }^{1)}$, Taku MASUYAMA2), Toshiyuki SHODA²), \\ Haruko KOIZUMI'), Masami SHINOHARA ${ }^{3)}$, \\ and Mutsuyoshi MATSUSHITA ${ }^{11}$
}
1)Japan Tobacco Inc., Central Pharmaceutical Research Institute, 1-1 Murasaki-cho, Takatsuki, Osaka 569-1125, 2)Japan Tobacco Inc., Central Pharmaceutical Research Institute, Toxicology Research Laboratories, 23 Naganuki, Hadano, Kanagawa 257-0024, ${ }^{3)}$ CLEA Japan Inc., Scientific Research Section, 1-2-7 Higashiyama, Meguro-ku, Tokyo 153-8533, Japan

\begin{abstract}
The spontaneously diabetic Torii (SDT) rat has recently been established as an animal model of non-obese type 2 diabetes, in which ocular complications severe occur. However, the function and morphological features of the diabetic renal lesions in SDT rats have not been reported in detail. Therefore, we evaluated changes over time in renal lesions in SDT rats. In addition, SDT rats were treated with insulin to observe whether these renal complications are caused by hyperglycemia. Renal functional parameters and renal lesions were monitored in SDT rats from 8 to 68 weeks of age. Sprague-Dawley (SD) rats of similar age were used as control animals. In the insulin-treated group of SDT rats, insulin pellets were implanted at 24 weeks of age to compare the development of renal lesions. The SDT rats began to develop hyperglycemia at 20 weeks of age. In the histopathological examination of the kidney, glycogen deposition of the renal tubular epithelium and renal tubular dilation were observed from 24 weeks of age in the untreated $S D T$ rats, and the changes in the renal tubules markedly progressed with aging. Moreover, thickening of the glomerular basement membrane was observed from 32 weeks of age. At 50 weeks of age, the glomeruli showed increase of mesangial matrix, with predominantly diffuse lesions showing by 68 weeks of age. The mesangial proliferation gradually progressed. In the SD rats, no renal lesions were present at 50 and 68 weeks of age. SDT rats with insulin treatment remained normoglycemic throughout observation and their renal functional parameters were normal. Glycemic control in SDT rats prevented the development of renal lesions. The features of SDT rats indicate their usefulness as an animal model for investigating diabetic nephropathy.
\end{abstract}

Key words: diabetes, diabetic nephropathy, histopathology, insulin, SDT rat 


\section{Introduction}

Human diabetes is a multifaceted disease, the complications of which have a profound impact on patient prognosis. Diabetic nephropathy is a serious complication of diabetes mellitus [8] making it essential to develop animal models of diabetic complications to simulate the pathogenesis in humans. The spontaneously diabetic Torii (SDT) rat is a new model for non-obese type 2 diabetes [13,24]. SDT rats are characterized by long survival despite persistent hyperglycemia when not treated with insulin, with a remarkably high survival rate of $92 \%$ at 65 weeks of age. The SDT rat is an ideal model for investigating diabetic complications with gradual progression and is the only diabetic model known with progression to proliferative retinopathy $[13,21]$.

Another complication of diabetes is diabetic nephropathy. It is caused by glomerulosclerosis, the pathogenesis of which is related to chronic hyperglycemia $[1,4,5]$. Patients with advanced diabetic nephropathy have few treatment options and often have a poor prognosis [19, 20]. In this study, we monitored pathobiochemical parameters and histopathological findings of kidneys in male SDT rats from 8 to 68 weeks of age. We also evaluated the effects of insulin implant in order to control blood glucose levels. Then we examined the rats to determine whether renal dysfunction in this model is caused by hyperglycemia. We found the SDT rat to be a useful animal model, because renal dysfunction of SDT rats had a tendency to be improved by insulin treatment.

\section{Materials and Methods}

\section{Animals}

Male SDT rats (CLEA Japan, Tokyo, Japan) were used from at 6 weeks of age (maximum of 68 weeks). Age-matched Sprague-Dawley (SD) rats (Crj: CD (SD); Charles River Japan, Yokohama, Japan) were maintained under similar conditions as control animals. In addition, at 24 weeks of age SDT rats were divided into untreated and insulin-treated groups. The rats were housed in a climate-controlled room with a temperature of $23 \pm 3^{\circ} \mathrm{C}$, humidity of $55 \pm 15 \%$, and a $12 \mathrm{~h}$ lighting cycle. Basal diet (CRF-1, Charles River Japan, Yokohama, Japan) and water were provided ad libitum. To control blood glucose levels in the insulin treated
SDT rats, an insulin pellet (Linshin Canada, Scarborough, Ontario, Canada) was implanted subcutaneously under light anesthesia with ether. Insulin was released at a maximum rate of $2 \mathrm{U} / 24 \mathrm{~h} / \mathrm{implant}$. Since the incidence of diabetes in the male SDT rat increases from about 20 weeks of age, blood glucose monitoring was started at 20 weeks of age. The blood glucose level was measured once weekly (9:00 to 11:00 AM), and if the blood glucose level of an insulin-treated SDT rat was $>14 \mathrm{mmol} / \mathrm{l}$, an additional insulin pellet was implanted. All the investigations were approved by the committee for the human care and use of animals of our laboratory, in accordance with the Standards Relating to the Care and Management of Experimental Animals (Notification No.6, March 27, 1980, of the Prime Minister's Office of Japan).

\section{Biological Parameters}

Biological parameters of untreated SDT and SD rats from 20 to 56 weeks of age were evaluated every 4 weeks. Blood samples were collected from the tail vein to measure glucose levels (hexokinase method). The insulin-treated SDT rat was evaluated in a similar manner from 36 weeks of age. Observation of blood glucose levels for insulin treatment was performed every week. Urine samples were collected by placing the animals in metabolic cages with feed and water for $24 \mathrm{~h}$. Urinary volume and protein were measured from 20 weeks of age, and urinary albumin was measured from 36 weeks of age. Measurements were performed with an automatic analyzer (Hitachi 7170S, Hitachi High-Technologies Co, Tokyo, Japan). Creatinine clearance ( $\mathrm{ml} / \mathrm{min})$ was calculated by dividing the 24-h urinary excretion of creatinine by the serum creatinine concentration.

\section{Tissue Sampling and Histopathology}

Necropsy was performed on untreated SDT rats and SD rats at $8,16,24,32,50$, and 68 weeks of age $(n=6$ for each age). In the SDT rats treated with insulin, necropsy was performed at 50 and 68 weeks of age (n $=6$ for both ages). Since a few animals died during this long experiment, satellite groups were prepared and six animals were secured for each age. All animals were sacrificed by exsanguination under light anesthesia with ether, and the kidneys were immediately sampled and fixed in 4\% paraformaldehyde. After resection, the tissue was paraffin-embedded by standard 
techniques and thin-sectioned ( 3 to $5 \mu \mathrm{m}$ ). The samples were stained with hematoxylin and eosin (HE), periodic acid Schiff (PAS), and Masson's trichrome (MT) stain. Immunohistochemical (IHC) staining was performed using a Nichirei Histofine kit (Nichirei Co., Tokyo, Japan). Anti-collagen type IV antibody (C-19; Santa Cruz Biotechnology, Inc., California, US) was diluted 1:100 and reacted overnight at $4^{\circ} \mathrm{C}$. The reaction was visualized with 3,3'-diaminobenzidine- $\mathrm{H}_{2} \mathrm{O}_{2}$ (DAB). The kidney samples were all examined histopathologically in a blind manner, and the findings were graded from normal (-) to severe (+3). A grade of + was used to represent focal or weakly positive sections, ++ was used to represent half of the section area positive, and +++ was used to represent more than half of the whole area positive in a tissue section. For the glomerular findings, more than one hundred glomeruli per animal were observed in this study.

\section{Statistical Analysis}

The results of the biological parameters are expressed as the mean \pm standard error. Statistical analysis of differences between mean values was performed using the F-test, followed by the Student's $t$-test or Aspin-Welch's $t$-test. Differences were defined as significant at $P<0.05$.

\section{Results}

\section{Biological Parameters}

In SDT rats, blood glucose levels were elevated at 20 weeks of age, with persistent hyperglycemia from 24 to 56 weeks of age $(36.2-54.1 \mathrm{mmol} / \mathrm{l})$ in the untreated animals. Blood insulin levels from 36 to 56 weeks of age $(0.44-1.22 \mathrm{ng} / \mathrm{ml})$ were lower in the untreated SDT rats compared to those of SD rats (4.36-5.78 ng/ml), and untreated SDT rats showed hyperglycemia with hypoinsulinemia. In the insulin-treated group of SDT rats, however, blood glucose levels did not rise. The levels were similar to those in SD rats (Fig. 1).

In untreated SDT rats, urinalysis showed an increase in urine volume and urinary protein at 24 weeks of age, and further increases thereafter. Urinary protein in untreated SDT rats increased from $29.4 \mathrm{mg} / 24 \mathrm{~h}$ to 318.6 $\mathrm{mg} / 24 \mathrm{~h}$. At 36 weeks of age and later, urinary albumin (12.9-35.5 mg/24h) was also higher compared to the SD rats $(2.6-8.4 \mathrm{mg} / 24 \mathrm{~h})$. In the insulin treated group,

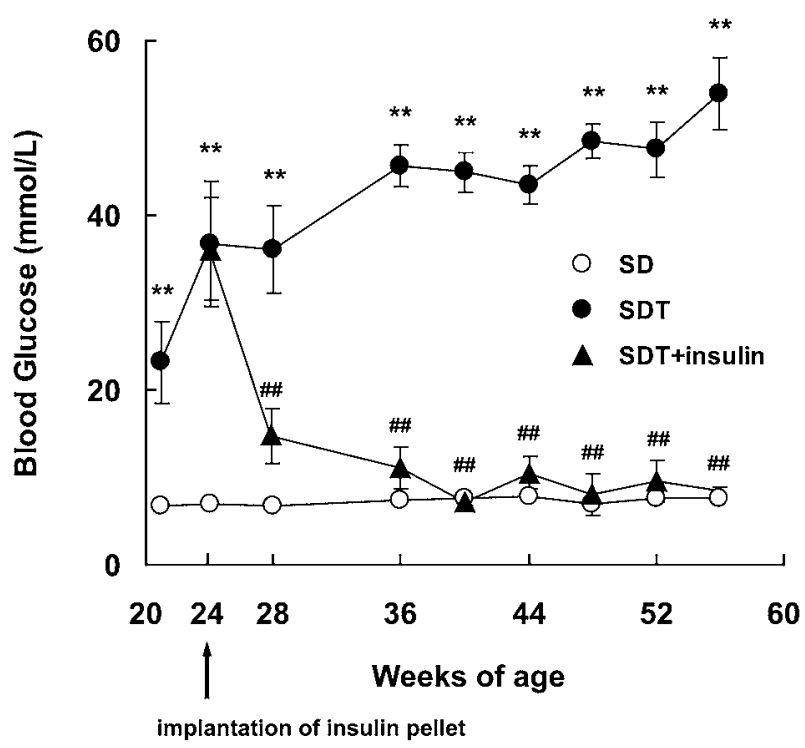

Fig. 1. Changes of blood glucose levels in SD, SDT and SDT+insulin rats. SDT rats showed persistent hyperglycemia and insulin treatment suppressed the hyperglycemia. Data represent means $\pm \mathrm{SE}$ (SD rat; $\mathrm{n}=6-8$, SDT rat; $\mathrm{n}=4-10$, SDT+insulin rat; $\mathrm{n}=3-9)$. $* * P<0.01$; significantly different from the SD rat. ${ }^{\#} P<0.01$; significantly different from the SDT rat.

the values for these urinary parameters were clearly decreased, and the changes were similar to those seen in SD rats (Fig. 2).

In untreated SDT rats, creatinine clearance tended to decrease at 20 and 24 weeks of age as compared with SD rats, but the levels of creatinine clearance were generally higher from 28 weeks of age. The reason why creatinine clearance in the SD rats greatly fluctuated is unknown. In the insulin-treated group, there was some variation with evaluation time point, but creatinine clearance was always lower than that of the untreated SDT rats (Fig. 2).

\section{Histopathological Analyses}

In the untreated SDT rats, histopathological examination of the kidneys revealed changes in the renal tubules from 24 weeks of age, and in the glomeruli, from 32 weeks of age (Table 1). In the kidneys of age-matched SD rats, there were no pathological findings.

In the glomeruli, basement membrane thickening was observed from 32 weeks of age, and the change progressed with aging. Mesangial matrix proliferation was observed from 50 weeks of age. These findings included 
A

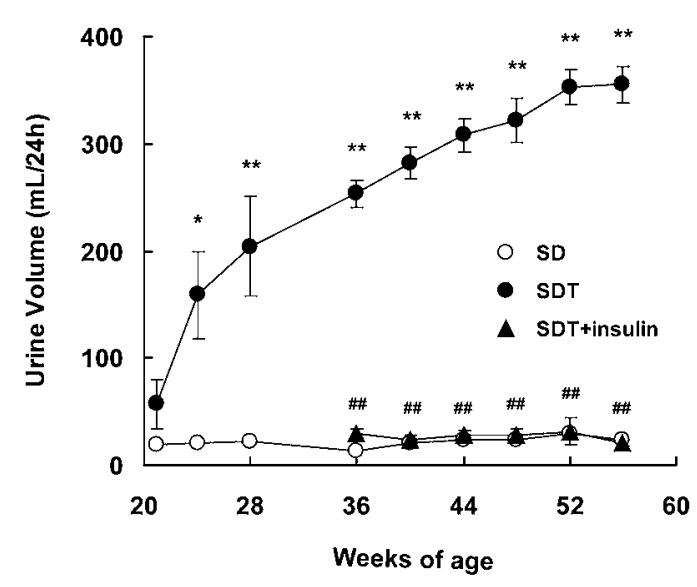

C

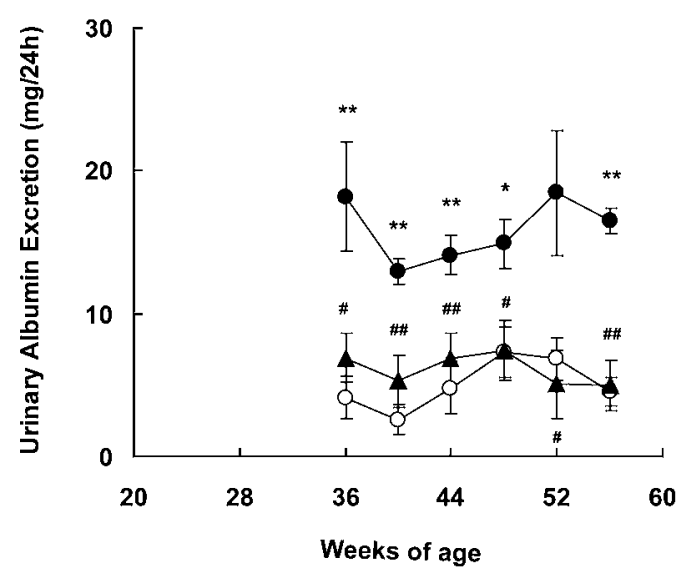

B

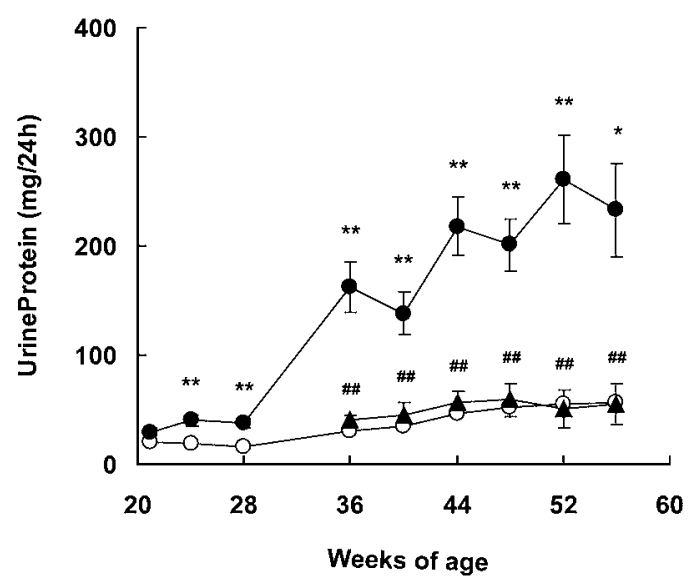

D

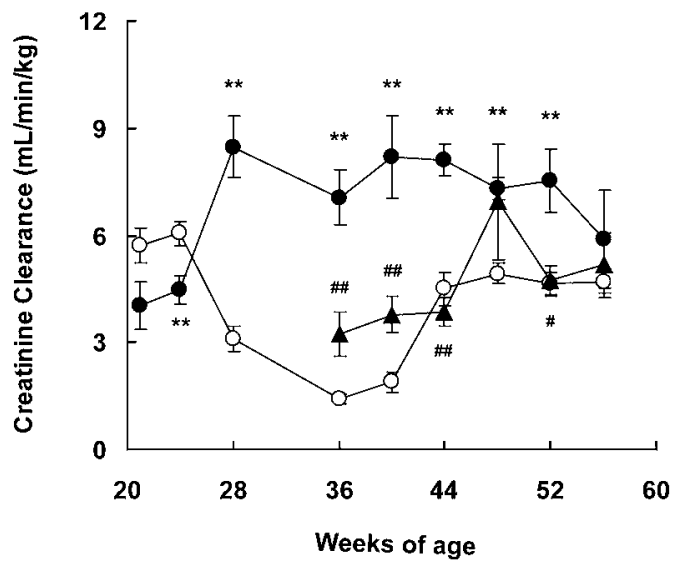

Fig. 2. Changes of biological parameters in SD, SDT and SDT+insulin rats. SDT rats showed deterioration of renal functional parameters and insulin treatment ameliorated the dysfunction. A: Urinary volume, B: Urinary protein, C: Urinary albumin, D: Creatinine clearance. Data represent means \pm SE (SD rat; $n=6-8$, SDT rat; $n=4-10$, SDT+insulin rat; $\mathrm{n}=3-9) . * P<0.05, * * P<0.01$; significantly different from the $\mathrm{SD}$ rat. ${ }^{\#} P<0.05,{ }^{*} P<0.01$; significantly different from the SDT rat.

an increase in PAS positive areas, fibrous proliferation shown by MT staining, and increased type IV collagen positive areas shown by IHC staining (Fig. 3-1). The glomerular changes gradually progressed with aging, with diffuse glomerular lesions seen at 50 and 68 weeks of age. At 68 weeks of age, nodular lesions were observed in a few glomerular capillary loops by PAS staining (Fig. 3-2). The nodular lesions showed weakly positive for MT staining and collagen type IV IHC staining.

In the renal tubules, glycogen deposition in the tubular epithelium (Armanni-Ebstein lesions) and tubular dilation were noted starting at 24 weeks of age in the untreated SDT rats (Table 1). There were increased hyaline casts at 50 weeks of age. These renal tubular changes markedly progressed with aging, and severe changes throughout the kidneys were present by 68 weeks of age. At 50 and 68 weeks of age, tubules with glycogen deposition were seen throughout the renal cortex. However, the insulin-treated group of SDT rats at 50 and 68 weeks of age showed no glomerular or tubular lesions (Fig. 3-1, Fig. 4). Renal damage in SDT rats was obviously prevented by insulin treatment.

\section{Discussion}

The SDT rat is a model of type 2 diabetes without 
Table 1. Histopathological findings of the kidney in SD, SDT and SDT+insulin rat

\begin{tabular}{|c|c|c|c|c|c|c|c|c|c|c|c|c|c|c|c|}
\hline \multirow{3}{*}{ Pathological findings } & \multicolumn{15}{|c|}{ weeks of age } \\
\hline & \multirow{2}{*}{$\begin{array}{l}8 \\
-\end{array}$} & \multirow{2}{*}{$\begin{array}{c}16 \\
-\end{array}$} & \multicolumn{2}{|c|}{24} & \multicolumn{3}{|c|}{32} & \multicolumn{4}{|c|}{50} & \multicolumn{4}{|c|}{68} \\
\hline & & & - & + & - & + & ++ & - & + & ++ & +++ & - & + & ++ & +++ \\
\hline \multicolumn{16}{|l|}{ SD } \\
\hline ND & 6 & 6 & 6 & & 6 & & & 6 & & & & 6 & & & \\
\hline \multicolumn{16}{|l|}{ SDT } \\
\hline Thickening of glomerular basement membrane & 6 & 6 & 6 & 0 & 2 & 4 & 0 & 0 & 3 & 3 & 0 & 0 & 2 & 4 & 0 \\
\hline Increased mesangial matrix in glomeruli & 6 & 6 & 6 & 0 & 6 & 0 & 0 & 0 & 2 & 4 & 0 & 0 & 2 & 4 & 0 \\
\hline Nodular lesion in glomeruli & 6 & 6 & 6 & 0 & 6 & 0 & 0 & 6 & 0 & 0 & 0 & 4 & 2 & 0 & 0 \\
\hline \multicolumn{16}{|l|}{ Glycogen accumulation/vacuolation } \\
\hline of renal tubular epithelium & 6 & 6 & 0 & 6 & 0 & 2 & 4 & 0 & 0 & 3 & 3 & 0 & 0 & 2 & 4 \\
\hline Dilatation of renal tubules & 6 & 6 & 0 & 6 & 0 & 3 & 3 & 0 & 1 & 4 & 1 & 0 & 0 & 5 & 1 \\
\hline Urinary cast in renal tubules & 6 & 6 & 6 & 0 & 6 & 0 & 0 & 0 & 2 & 4 & 0 & 0 & 1 & 5 & 0 \\
\hline \multicolumn{16}{|l|}{ SDT + insulin } \\
\hline ND & $\mathrm{NE}$ & $\mathrm{NE}$ & & $\mathrm{E}$ & & $\mathrm{NE}$ & & 6 & & & & 6 & & & \\
\hline
\end{tabular}

Each of the findings were qualitatively divided into three grades from + to +++ , slight, moderate and severe. NE: Not examined. ND: Not detected.

obesity, in which hyperglycemia spontaneously develops, predominantly due to an insulin secretory defect resulting from pathological damage to the pancreatic islets, especially $\beta$-cells [13]. In addition to marked hyperglycemia, severe ocular complications are a characteristic of SDT rats [21].

Diabetic nephropathy is usually asymptomatic in its early stages but can have a profound impact on patients who later develop renal failure. The underlying pathogenesis in diabetic nephropathy is mainly related to glomerulosclerosis $[4,5]$, but in human diabetes, extraglomerular lesions such as arteriolar sclerosis and tubulointerstitial degeneration and fibrosis can exacerbate glomerular injury [1, 2, 4, 5, 19].

In our study, the SDT rats were hyperglycemic and already had renal lesions at 24 weeks of age. Since creatinine clearance in untreated SDT rats was elevated from 28 to 52 weeks of age compared to SD rats (Fig. $2 \mathrm{D})$, renal hyperfiltration in SDT rats was suggested during this period. The renal lesions were broadly divided into glomerulosclerotic changes with mesangial proliferation and basement membrane thickening, and renal tubular changes with glycogen deposition and tubular dilation. Changes similar to those we report in the renal tubules and glomeruli of SDT rats have also been reported in diabetic models, such as Wistar Bonn/Kobori (WBN) rats, Zucker diabetic fatty (ZDF) rats and GotoKakizaki (GK) rats. In WBN rats, only males spontaneously develop hyperglycemia, glycosuria, hypoinsulinemia and glucose intolerance from about 9 months of age. As for renal lesions, the urinary total protein begins to increase at about 13 months of age in WBN rats and reaches $50-300 \mathrm{mg} / 24 \mathrm{~h}$ at $13-28$ months of age, which is significantly higher than in age-matched male Wistar rats. Renal tubular change with glycogen deposition is observed from 12 months of age, and thickening of the basement membrane and increase of mesangial matrix are seen in the glomeruli from about 17 months of age $[10,16]$. Moreover, the incidence and degree of glycogen accumulation in renal tubules and the incidence of mesangial matrix in glomeruli shows good correlation with the blood glucose concentration of WBN rats $[25,26]$. The ZDF rat is a model of type II diabetes based on impaired glucose tolerance caused by the inherited insulin-resistance gene $f a$, and shows overt diabetes despite hyperinsulinemia as early as 3 months of age. Blood glucose levels increase of the ZDF rat further with a considerable decrease of insulin levels at 5 months. The glomerular filtration rate is elevated until 3 months, but falls to the level seen in lean rats by 7 months. Glomerulosclerosis occurs as early as 5 months of age and tends to increase with age. The earliest glomerular changes in ZDF rats are glomerular enlargement with mild mesangial widening which are found as early as 5 months of age. In addition, ZDF rats exhibit hydropic degeneration of renal tubules $[7,23]$. In 


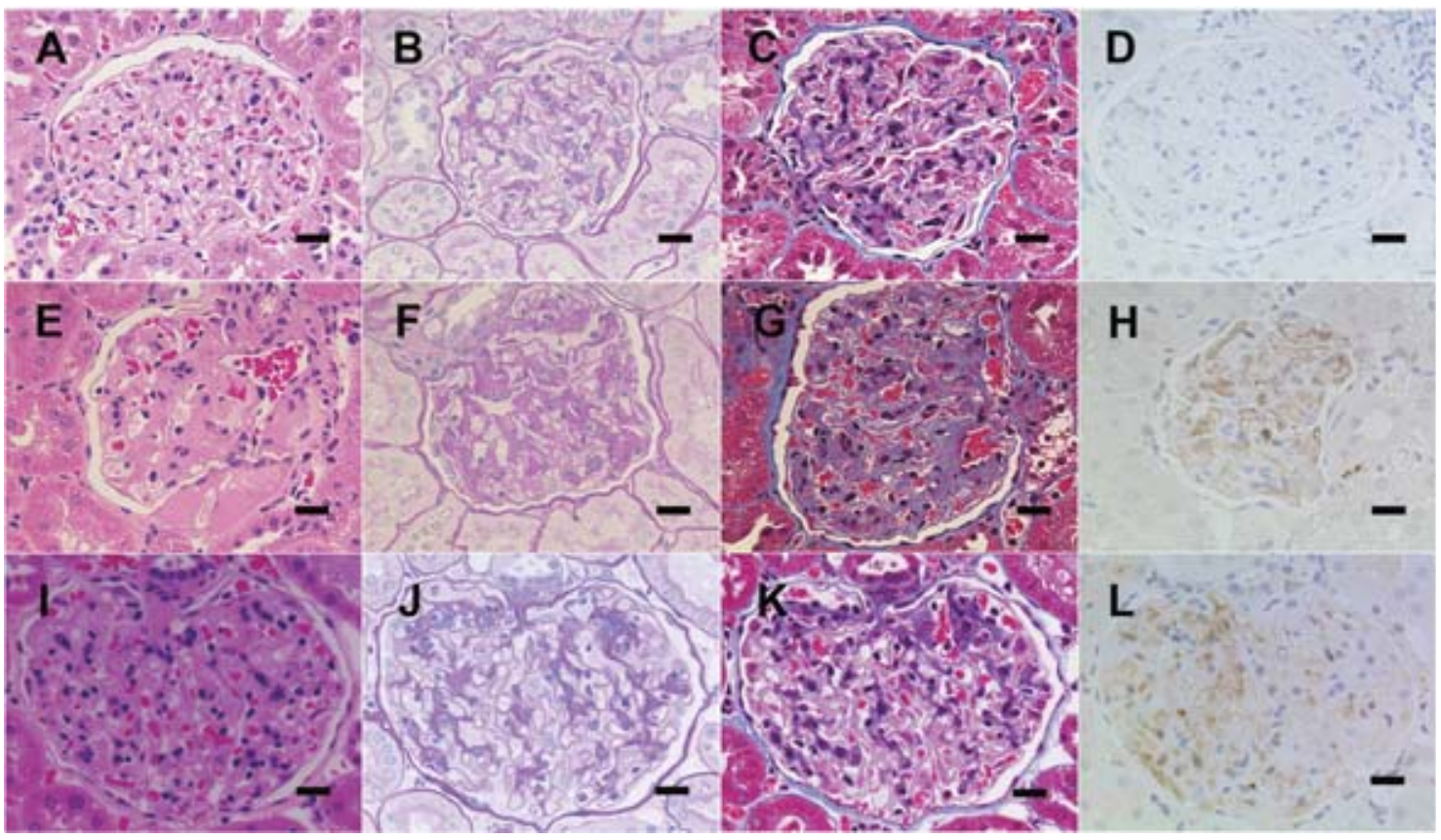

Fig. 3-1. Histological and immunohistological analysis of glomeruli in SD, SDT and SDT+insulin rats. In the glomeruli of SDT rats, basement membrane thickening and mesangial matrix proliferation were observed at 50 weeks of age. SDT rats with insulin treatment showed improvement of the glomerular changes. A, E and I: HE stained sections; B, F and J: PAS stain; C, G and K: MT stain; D, H and L: Collagen type IV immunohistostained sections. Kidney sections are from SD $(\mathrm{A}-\mathrm{D})$ and SDT rats $(\mathrm{E}-\mathrm{H})$ at 50 weeks of age. SDT rats treated with insulin pellets (I-L) at 68 weeks of age. $\mathrm{Bar}=20 \mu \mathrm{m}$.

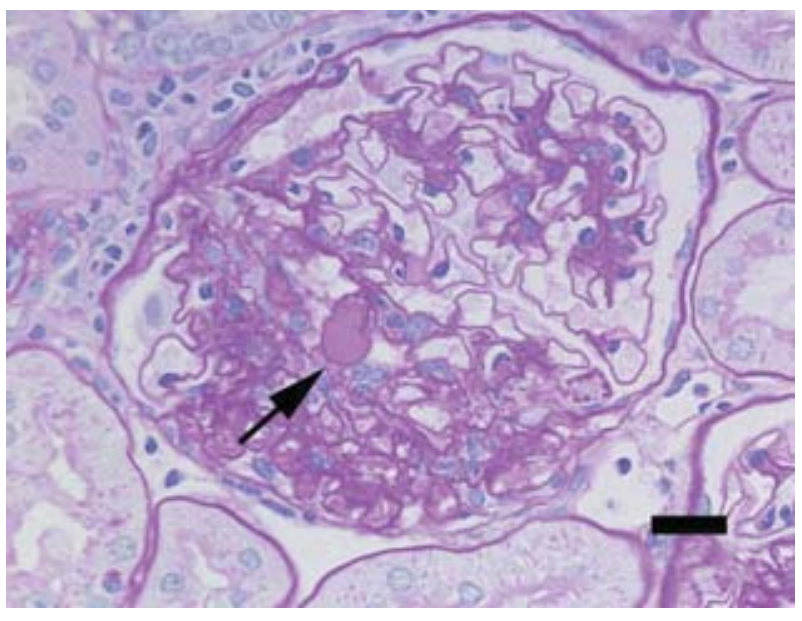

Fig. 3-2. Representative microphotograph of a glomerulus found in the kidney of a SDT rat at 68 weeks of age. PAS stain. $\mathrm{Bar}=20 \mu \mathrm{m}$. Nodular lesions were observed in a few glomeruli (Arrow).
Goto-Kakizaki (GK) rats, a genetic model of non-obese type II diabetes, glucose stimulated insulin secretion is reduced and glucose tolerance is impaired. Moderate hyperglycemia is sustained in GK rats for a lifetime. The excretion of urinary proteins in GK rats has a tendency to increase at 6-24 month of age, accompanied by renal histologic abnormalities such as focal glomerulosclerosis, mesangial matrix expansion, and thickening of basement membranes [17, 22]. Also, the vacuolation of renal tubular epithelium has been observed in GK rats [18]. Nodular lesions as shown in untreated SDT rats (Fig. 3-2) were not observed in the above-mentioned diabetic rats. Findings indicating renal failure in untreated SDT rats were not significantly observed during 20-68 weeks of age. Moreover, the relationship between progression of diabetic nephropathy and mortality in SDT rats was not examined. In the future, these topics should be investigated using rats, older than 70 weeks of age.

In the course of the experiment, basement membrane thickening and mesangial proliferation were first ob- 

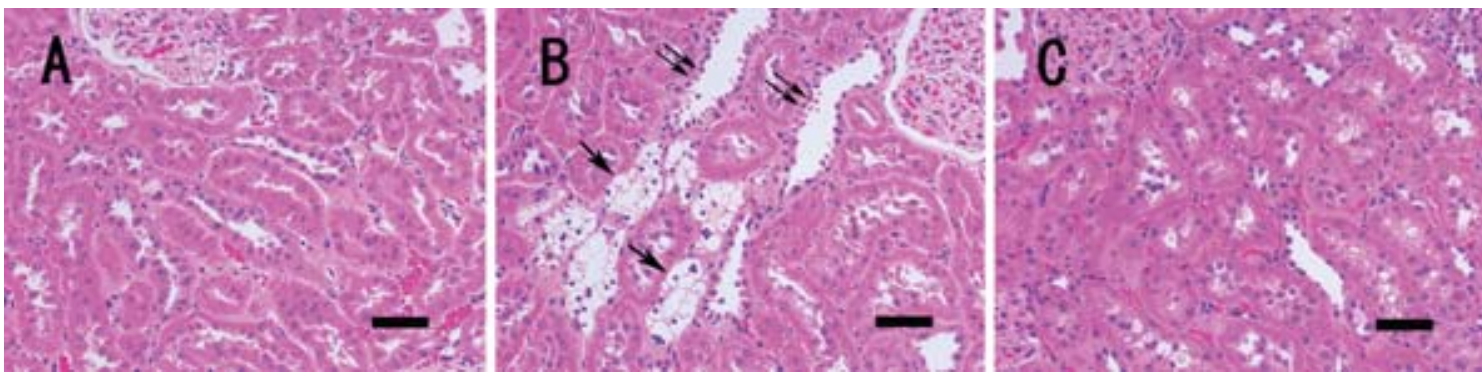

Fig. 4. HE-stained representative sections of the kidneys of a SD rat (A), SDT rat (B) and SDT rat treated with insulin pellets (C) at 68 weeks of age. Bar $=50 \mu \mathrm{m}$. In the renal tubules of SDT rats, glycogen deposition in the tubular epithelium (Single arrow) and tubular dilation (Double arrow) were observed. SDT rats with insulin treatment showed amelioration of the tubular changes.

served in the glomeruli of the untreated SDT rats. These findings were followed by thickening of the capillary loops and diffuse glomerular lesions. In humans, the histopathologic features of glomerular lesions are generally divided into 3 types: diffuse lesions, nodular lesions, and exudative lesions [9]. Of these, the predominant changes in long-standing diabetes are diffuse lesions [9]. In the untreated SDT rats, the onset and progression of diffuse lesions was similar to that in human diabetes. The extent of the progression of glomerular lesions was similar to that seen in human diabetic nephropathy in type 2 or early type 3 . The histopathological findings correlated with increases in urinary protein and albumin excretion, and these increases were probably related to the increase in glomerular pressure which is seen early after diabetes onset $[14,15]$ and disruption of the charge barrier causing by a decrease in negatively charged substances [3]. Another influential factor may be increased GBM pore size, as reported in a study using STZ-induced diabetic rats [11].

In the renal tubules of untreated SDT rats, glycogen deposition in the epithelial cells was similar to the socalled Armanni-Ebstein lesions in human diabetic nephropathy [12]. Glycogen accumulates as a result of increased resorption of glucose in the proximal tubules [12]. The absence of similar glycogen accumulation in the insulin-treated group, in which glucose was wellcontrolled, suggests reactive changes due to fluctuations in glucose. In addition, dilation of the tubular lumen in the untreated SDT rats was predominantly in the distal tubules. This change, which is probably related to increased urine volume, was not seen in the insulin-treated group, in which urine volume was normal. Thus, the histological findings in the untreated SDT rats correlated with the changes in renal functional parameters. In advanced diabetic nephropathy in humans, severe interstitial lesions such as cellular infiltration and fibrosis accompanied by narrowing tubular lumen and diminished tubular epithelium have been reported [6]. Similar lesions, however, were not seen in the untreated SDT rats of this study.

In the insulin-treated group of SDT rats, there was clear improvement in all of the above kidney related parameters. This further demonstrates that the renal lesions in SDT rats are dependent on hyperglycemia. The histopathological findings in the kidneys of the untreated SDT rats were correlated with blood glucose levels and each urinalysis parameter. Improvement of renal lesion by long-term glucose control has also been reported in other diabetic models. GK rats exhibited slight damage to the glomerulus and renal tubule, which was improved by glycemic control [18]. Moreover, ZDF rats exhibited moderate renal lesions, including increased mesangial matrix, glomerular hyaline droplets and hydropic degeneration of renal tubules [23], which were also improved by glycemic control.

In conclusion, SDT rats are a useful model for investigating complications of diabetes. Use of this rat model will assist in the further elucidation of the pathogenesis of human diabetic nephropathy and in discovering new drugs.

\section{Acknowledgment(s)}

We wish to thank Mr. Hiroyuki Hirayama and Mr. Hiroyuki Kanada (JT Creative Service) for long-term animal care. 


\section{References}

1. Andersen, A.R., Christiansen, J.S., Andersen, J.K., Kreiner, S., and Deckert, T. 1983. Diabetic nephropathy in Type 1 (insulin-dependent) diabetes: an epidemiological study. Diabetologia 25: 496-501.

2. Dalla Vestra, M., Saller, A., Mauer, M., and Fioretto, P. 2001. Role of mesangial expansion in the pathogenesis of diabetic nephropathy. J. Nephrol. 14 (Suppl. 4): S51-57.

3. Deckert, T., Feldt-Rasmussen, B., Djurup, R., and Deckert, M. 1988. Glomerular size and charge selectivity in insulindependent diabetes mellitus. Kidney Int. 33: 100-106.

4. Gellman, D.D., Pirani, C.L., Soothill, J.F., Muehrcke, R.C., and Kark, R.M. 1959. Diabetic nephropathy: a clinical and pathologic study based on renal biopsies. Medicine (Baltimore) 38: 321-367.

5. Gellman, D.D., Pirani, C.L., Soothill, J.F., Muehrcke, R.C., Maduros, W., and Kark, R.M. 1959. Structure and function in diabetic nephropathy; the importance of diffuse glomerulosclerosis. Diabetes 8: 251-256.

6. Gilbert, R.E. and Cooper, M.E. 1999. The tubulointerstitium in progressive diabetic kidney disease: more than an aftermath of glomerular injury? Kidney Int. 56: 1627-1637.

7. Hoshi, S., Shu, Y., Yoshida, F., Inagaki, T., Sonoda, J., Watanabe, T., Nomoto, K., and Nagata, M. 2002. Podocyte injury promotes progressive nephropathy in zucker diabetic fatty rats. Lab. Invest. 82: 25-35.

8. Hostetter, T.H. 1992. Diabetic nephropathy. Metabolic versus hemodynamic considerations. Diabetes Care 15: 1205-1215.

9. Inomat, S., Haneda, M., Moriya, T., Katayama, S., Iwamoto, Y., Sakai, H., Tomino, Y., Matsuo, S., Asano, Y., and Makino, H. 2005. Revised criteria for the early diagnosis of diabetic nephropathy. Nippon Jinzo Gakkai Shi 47: 767-769 (in Japanese with English summary).

10. Ishizaki, M., Masuda, Y., Fukuda, Y., Yamanaka, N., Masugi, Y., Shichinohe, K., and Nakama, K. 1987. Renal lesions in a strain of spontaneously diabetic WBN/Kob rats. Acta Diabetol. Lat. 24: 27-35.

11. Isogai, S., Mogami, K., Shiina, N., and Yoshino, G. 1999. Initial ultrastructural changes in pore size and anionic sites of the glomerular basement membrane in streptozotocininduced diabetic rats and their prevention by insulin treatment. Nephron 83: 53-58.

12. Kang, J., Dai, X.S., Yu, T.B., Wen, B., and Yang, Z.W. 2005. Glycogen accumulation in renal tubules, a key morphological change in the diabetic rat kidney. Acta Diabetol. 42: 110-116.

13. Masuyama, T., Komeda, K., Hara, A., Noda, M., Shinohara, M., Oikawa, T., Kanazawa, Y., and Taniguchi, K. 2004. Chronological characterization of diabetes development in male Spontaneously Diabetic Torii rats. Biochem. Biophys. Res. Commun. 314: 870-877.
14. Mathiesen, E.R., Ronn, B., Jensen, T., Storm, B., and Deckert, T. 1990. Relationship between blood pressure and urinary albumin excretion in development of microalbuminuria. Diabetes 39: 245-249.

15. Mogensen, C.E. 1986. Early glomerular hyperfiltration in insulin-dependent diabetics and late nephropathy. Scand. J. Clin. Lab. Invest. 46: 201-206.

16. Mori, Y., Yokoyama, J., Nishimura, M., Oka, H., Mochio, S., and Ikeda, Y. 1992. Development of diabetic complications in a new diabetic strain of rat (WBN/Kob). Pancreas 7: 569-577.

17. Nobrega, M.A., Fleming, S., Roman, R.J., Shiozawa, M., Schlick, N., Lazar, J., and Jacob, H.J. 2004. Initial characterization of a rat model of diabetic nephropathy. Diabetes 53: 735-742.

18. Ohta, T., Miyajima, K., Komuro, G., Furukawa, N., and Yonemori, F. 2003. Antidiabetic effect of chronic administration of JTT-608, a new hypoglycemic agent, in diabetic GotoKakizaki rats. Eur. J. Pharmacol. 476: 159-166.

19. Raptis, A.E. and Viberti, G. 2001. Pathogenesis of diabetic nephropathy. Exp. Clin. Endocrinol. Diabetes 109 (Suppl. 2): S424-437.

20. Rudberg, S., Persson, B., and Dahlquist, G. 1992. Increased glomerular filtration rate as a predictor of diabetic nephropathy-an 8-year prospective study. Kidney Int. 41: 822-828.

21. Sasase, T., Ohta, T., Ogawa, N., Miyajima, K., Ito, M., Yamamoto, H., Morinaga, H., and Matsushita, M. 2006. Preventive effects of glycaemic control on ocular complications of Spontaneously Diabetic Torii rat. Diabetes Obes. Metab. 8: 501-507.

22. Sato, N., Komatsu, K., and Kurumatani, H. 2003. Late onset of diabetic nephropathy in spontaneously diabetic GK rats. Am. J. Nephrol. 23: 334-342.

23. Shibata, T., Takeuchi, S., Yokota, S., Kakimoto, K., Yonemori, F., and Wakitani, K. 2000. Effects of peroxisome proliferator-activated receptor-alpha and -gamma agonist, JTT-501, on diabetic complications in Zucker diabetic fatty rats. Br. J. Pharmacol. 130: 495-504.

24. Shinohara, M., Masuyama, T., Shoda, T., Takahashi, T., Katsuda, Y., Komeda, K., Kuroki, M., Kakehashi, A., and Kanazawa, Y. 2000. A new spontaneously diabetic nonobese Torii rat strain with severe ocular complications. Int. J. Exp. Diabetes Res. 1: 89-100.

25. Tago, Y., Katsuta, O., Tsuchitani, M., Narama, I., and Itakura, C. 1991. Glomerular lesions in spontaneously occurring diabetic WBN/Kob rats. J. Comp. Pathol. 104: 367-377.

26. Tsuchitani, M., Kuroda, J., Nagatani, M., Miura, K., Katoh, T., Saegusa, T., Narama, I., and Itakura, C. 1990. Glycogen accumulation in the renal tubular cells of spontaneously occurring diabetic WBN/Kob rats. J. Comp. Pathol. 102: 179-190. 